\title{
Macrófagos e sua relação com o carcinoma de células escamosas Oral
}

\author{
Macrophages and their Relationship with Oral Squamous Cell Carcinoma
}

\author{
Rafaella Bastos Leite \\ Mestre - Programa de Pós-Graduação em Odontologia - Universidade \\ Estadual da Paraíba - UEPB - Campina Grande - PB - Brasil. \\ Hellen Bandeira de Pontes Santos \\ Mestranda - Programa de Pós-Graduação em Odontologia - Universidade \\ Estadual da Paraíba - UEPB - Campina Grande - PB - Brasil. \\ Priscilla Suassuna Carneiro lúCiO \\ Mestre - Programa de Pós-Graduação em Odontologia - Universidade \\ Estadual da Paraíba - UEPB - Campina Grande - PB - Brasil. \\ Pollianna Muniz ALVES \\ Professor Doutor - Programa de Pós-Graduação em Odontologia - Universidade \\ Estadual da Paraíba - UEPB - Campina Grande - PB - Brasil. \\ Gustavo PINA GODOY \\ Professor Doutor - Programa de Pós-Graduação em Odontologia - Universidade \\ Estadual da Paraíba - UEPB - Campina Grande - PB - Brasil. \\ Cassiano Francisco Weege nONAKa \\ Professor Doutor - Programa de Pós-Graduação em Odontologia - Universidade \\ Estadual da Paraíba - UEPB - Campina Grande - PB - Brasil.
}

\section{RESUMO}

Os macrófagos constituem uma população heterogênea de células mieloides inatas que exibem considerável plasticidade. Estas células, que desempenham papéis importantes no combate às infecções e na resolução dos processos inflamatórios, representam um componente significativo do infiltrado inflamatório de vários tumores. Em virtude de sua capacidade de liberação de fatores angiogênicos, proteases e citocinas, os macrófagos associados a tumores (MATs) têm sido implicados na progressão de diversas neoplasias, incluindo o carcinoma de células escamosas (CCE) oral. Objetivo: realizar uma revisão da literatura sobre a origem dos macrófagos, seus principais aspectos fisiológicos e discutir sua relação com o CCE oral. Metodologia: foi realizada uma busca eletrônica na base de dados PubMed, selecionando os principais artigos da literatura em língua inglesa relacionados ao tema, publicados entre janeiro de 2000 e janeiro de 2015. Conclusão: os macrófagos constituem um componente importante do microambiente do CCE oral. A maior quantidade de MATs, em especial o fenótipo M2, pode estar associada a um comportamento biológico mais agressivo do CCE oral e, consequentemente, com a diminuição da sobrevida dos pacientes. Em decorrência do número relativamente pequeno de estudos sobre o assunto, pesquisas ainda são necessárias para esclarecer os mecanismos moleculares pelos quais os macrófagos são capazes de influenciar o comportamento biológico do CCE oral.

Palavras-chave: macrófagos; carcinoma de células escamosas; imuno-histoquímica.

\begin{abstract}
Macrophages are a heterogeneous population of innate myeloid cells that exhibit considerable plasticity. These cells, which play important roles in infection control and resolution of inflammatory processes, represent a significant component of the inflammatory infiltrate of many tumors. Due to their ability to release angiogenic factors, proteases, and cytokines, tumor-associated macrophages (TAMs) have been implicated in the progression of several malignancies, including oral squamous cell carcinoma (SCC). Aim: To perform a literature review regarding the origin of macrophages, their main physiological aspects and to discuss their
\end{abstract}


Rafaella Bastos Leite et al.

relationship with oral SCC. Methodology: An electronic search was performed on the PubMed database, selecting the main papers in English literature related to the subject, published between January 2000 and January 2015. Conclusion: Macrophages are an important component of the microenvironment of oral SCC. The largest amount of TAMs, specially M2 phenotype, may be associated with a more aggressive biological behavior of oral SCC and, consequently, decreased patient survival. Due to the relatively small number of studies on the subject, more investigations are needed to clarify the molecular mechanisms by which macrophages are able to influence the biological behavior of oral SCC.

Keywords: macrophages; squamous cell carcinoma; immunohistochemistry.

\section{INTRODUÇÃo}

Os macrófagos constituem uma população heterogênea de células mieloides inatas que residem em diversos tecidos, nos quais desempenham papéis cruciais no combate às infecções, na resolução dos processos inflamatórios e na modulação da resposta metabólica ao estresse tecidual., ${ }^{1,2,3}$ Para desenvolver estas respostas, os macrófagos exibem considerável plasticidade $\mathrm{e}$, em decorrência desta característica, estas células têm sido implicadas em processos patológicos diversos, como doenças metabólicas ${ }^{2}$ e neoplasias malignas, incluindo o carcinoma de células escamosas (CCE) oral. $4,5,6,7,8,9$

Dois estados de polarização relativamente distintos têm sido reconhecidos para essas células: macrófagos classicamente ativados (M1) e macrófagos ativados de forma alternativa (M2). Macrófagos M1 desenvolvemse em resposta a produtos microbianos e citocinas Th1, como interferon- $\gamma$ (IFN- $\gamma$ ) e fator de necrose tumoral- $\alpha$ (TNF- $\alpha$ ), ${ }^{4,7,8}$ e são considerados potentes células efetoras na eliminação de micro-organismos e células tumorais. ${ }^{4}$ Por outro lado, os macrófagos M2 podem se desenvolver em resposta a diversos estímulos, com destaque para as citocinas Th2, como a interleucina-4 (IL-4) e a interleucina-13 (IL-13), ${ }^{6}$ e desempenham funções importantes na regulação da resposta imune e na remodelação tecidual. , $^{1,3,10,11}$

Tumores sólidos são infiltrados por células inflamatórias e um número crescente de resultados oriundos de estudos em neoplasias diversas têm demonstrado uma estreita correlação entre o número de macrófagos nestes infiltrados e seu prognóstico. ${ }^{1,3,5,6,7,7,8} \mathrm{Com}$ base nestes achados, tanto o recrutamento quanto a ativação dos macrófagos associados a tumores (MATs) têm sido considerados elementos importantes na progressão tumoral ${ }^{4}$

Além disso, em virtude das diversas interações que podem ocorrer entre células neoplásicas e macrófagos ao longo do desenvolvimento e progressão tumorais, estudos têm sugerido que os MATs podem constituir alvos potenciais no tratamento de neoplasias malignas..$^{1,3,11}$ Dentre as estratégias terapêuticas descritas na literatura, destacamse o bloqueio do recrutamento e diferenciação dos macrófagos, a eliminação dos macrófagos do microambiente tumoral, a repolarização dos MATs e a inibição das funções dos MATs M2. ${ }^{1,11,12}$

Sendo assim, este trabalho tem o objetivo de realizar uma revisão da literatura sobre a origem dos macrófagos, seus principais aspectos fisiológicos, bem como discutir o papel destes tipos celulares no desenvolvimento e progressão do CCE oral, ampliando a compreensão sobre o assunto.

\section{Metodologia}

Foi realizada a busca eletrônica de publicações na base de dados PubMed, utilizando-se as seguintes palavras-chave, obtidas de acordo com o Medical Subject Headings (MeSH): macrophages, neoplasms, squamous cell carcinoma, oral cavity, tumor microenvironment e immunohistochemistry, com o operador booleano "AND". 
Foram adotados como critérios de inclusão dos estudos: a) reportar-se aos MATs, tratar de seus aspectos morfofisiológicos e imunohistoquímicos e/ou relacionar sua participação no desenvolvimento/progressão do CCE oral; b) ter sido desempenhado em modelo de estudo in vivo ou in vitro; c) ter sido divulgado no período de janeiro de 2000 a janeiro de 2015. Como critérios de exclusão, utilizouse qualquer outra língua que não a inglesa e artigos não relacionados ao tema. Segundo os critérios de inclusão e exclusão, realizou-se a seleção de 63 artigos que, após análise tiveram 33 excluídos. Ao final, foram selecionados trinta trabalhos publicados de maior relevância sobre o tema (Figura 1).

\section{REVISÃo dE LITERATURA}

\section{Origem}

Macrófagos estão presentes em quase todos os tecidos e desempenham um papel

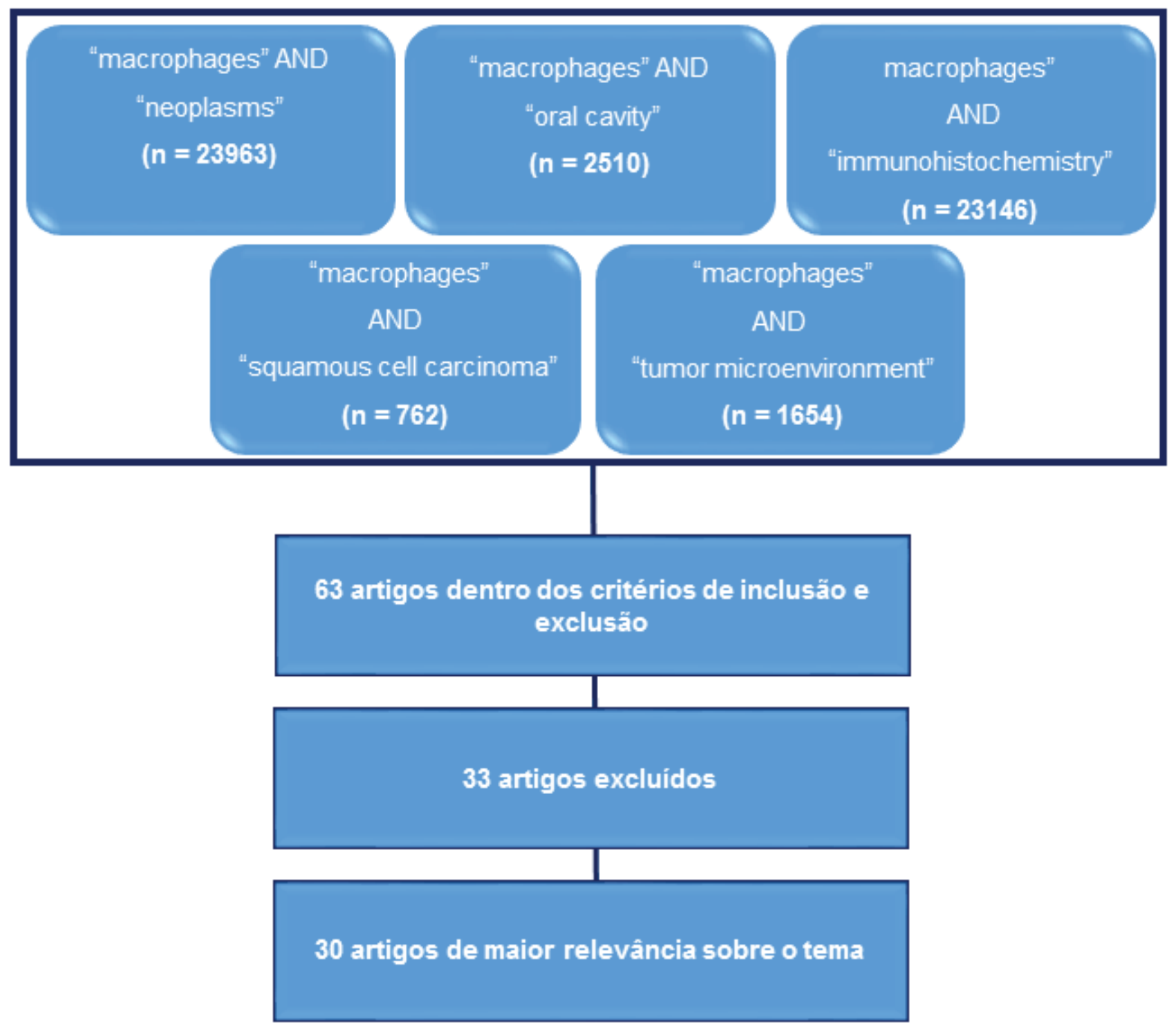

Figura 1- Critérios adotados para a inclusão e exclusão de artigos de revisão 
importante na manuteção da homeostase tecidual. Em adultos, células progenitoras mieloides dão origem aos monócitos do sangue periférico, os quais, por sua vez, se diferenciam em macrófagos. As células progenitoras mieloides são identificadas, na medula óssea, como unidades formadors de colônias granulócito/macrófago (GM-CFUs). Em resposta ao fator de formação de colônias de macrófagos, as GM-CFUs dão origem, em sequência, a unidades formadoras de colônias de macrófagos (M-CFUs), monoblastos e prómonócitos. Posteriormente, os pró-monócitos migram para o sangue periférico e diferenciamse em monócitos. Finalmente, os monócitos migram para diferentes tecidos e reconstituem as populações de longa duração tecidoespecíficas, tais como macrófagos alveolares e células de Kupffer. ${ }^{1,3}$

No entanto, evidências sugerem que nem todos os macrófagos teciduais têm origem a partir da diferenciação de monócitos do sangue periférico. Algumas populações de macrófagos residentes em tecidos, como as células de Langerhans na pele e da microglia no cérebro, parecem ser mantidas pela proliferação local. ${ }^{3}$

\section{Aspectos Fisiológicos}

Os macrófagos possuem uma vasta gama de receptores de superfície celular que são capazes de reconhecer tanto padrões moleculares associados a patógenos (PAMPs) quanto padrões moleculares associados ao dano (DAMPs), como receptores Toll-like, receptores $N O D$-like, receptores scavenger e lectinas. ${ }^{13}$ Dependendo da natureza do estímulo, os macrófagos podem produzir uma variedade de citocinas e quimiocinas pró-inflamatórias que recrutam, a partir da circulação, subpopulações celulares específicas do sistema imune para o local da injúria ou infecção. ${ }^{13,14}$ Por outro lado, em estágios tardios do reparo, os macrófagos podem assumir um fenótipo capaz de estimular o crescimento celular, realizar a fagocitose de células apoptóticas e, por fim, promover a resolução do processo inflamatório. ${ }^{14}$ Esta habilidade extraordinária de assumir fenótipos funcionais diversos permite que os macrófagos coordenem várias respostas a desafios ambientais. ${ }^{14}$

Embora apresentem considerável plasticidade funcional, dois estados de polarização relativamente distintos têm sido reconhecidos para estas células: macrófagos classicamente ativados (M1) e macrófagos ativados de forma alternativa (M2). Macrófagos M1 desenvolvem-se em resposta a citocinas Th1, como IFN-y e TNF- $\alpha$, e a produtos microbianos. Estas células são caracterizadas por: alta capacidade de apresentação de antígenos; alta produção de interleucina-12 (IL12) e interleucina-23 (IL-23); alta produção de intermediários tóxicos, como o óxido nítrico e espécies reativas de oxigênio; e alta expressão do antígeno leucocitário humano (HLA)-DR. 4,7

Por outro lado, os macrófagos M2 desenvolvem-se em resposta a diversos estímulos, com destaque para as citocinas Th2, ${ }^{7}$ e podem ser subdivididos nas subpopulações M2a, M2b e M2c ${ }^{3}$. Macrófagos M2a são estimulados pela IL-4 e pela IL-13, ao passo que macrófagos $M 2 b$ são induzidos por complexos imunes, lipopolissacarídeos, receptores Toll-like e pelo antagonista do receptor da interleucina-1 (IL-1ra). Por sua vez, a subpopulação M2c é induzida pela interleucina-10 (IL-10), pelo fator transformante de crescimento beta (TGF- $\beta$ ) e por glicocorticoides. ${ }^{3}$ Os macrófagos M2 apresentam grande capacidade de síntese de interleucina-10 (IL-10), fatores angiogênicos, como o fator de crescimento vascular endotelial (VEGF), metaloproteinases de matriz (MMPs)e moléculas de superfície, como CD204, CD206 e CD163. Tais células desempenham funções imunorreguladoras, promovem o reparo e a remodelação tecidual e têm sido implicadas na progressão de diversos tumores. , $^{1,10}$ 


\section{Macrófagos associados a tumores e o CARCINOMA DE CÉLULAS ESCAMOSAS ORAL}

No contexto dos MATs, pesquisas têm sugerido que a subpopulação M1 é capaz de destruir células neoplásicas, ao passo que a subpopulação M2 pode contribuir para a progressão tumoral. ${ }^{1,3}$ Os mecanismos pelos quais os MATs M2 auxiliam no crescimento e na disseminação tumoral ainda não são completamente conhecidos, mas investigações têm destacado a modulação da proliferação celular, da angiogênese, da remodelação da matriz extracelular (MEC) e da resposta imune. $^{1}$

Esta subpopulação celular expressa baixos níveis de IL-12, capaz de ativar e potencializar as funções citolíticas das células T CD8+e natural killer (NK), bem como altos níveis de IL-10, capaz de inibir a síntese de citocinas importantes para a ativação de células T. ${ }^{15}$ Uma das características mais importantes dos MATs M2 é sua capacidade de influenciar o crescimento tumoral por meio do estímulo à sobrevida e à invasão das células neoplásicas. ${ }^{4}$ Um dos mecanismos mais bem estabelecidos pelos quais os MATs são capazes de promover o desenvolvimento de tumores sólidos é a síntese de fatores que estimulam a proliferação e a migração das células neoplásicas, como o fator de crescimento epidérmico (EGF)..$^{1,11}$

Além disso, os MATs são capazes de regular a composição e a estrutura da MEC por meio da deposição de proteínas fibrosas, como quatro formas de colágeno, e da degradação destas e de outros componentes por meio da secreção de metaloproteinases, como as MMPs -1, -2 e -9, catepsinas, ativador do plasminogênio tipo uroquinase e ativador do plasminogênio tipo tecidual. 1,16,17

Embora as células $T$ regulatórias (Treg) tenham sido historicamente implicadas como os principais elementos responsáveis pela modulação da resposta imune em neoplasias, pesquisas recentes têm demonstrado que os MATs podem ser importantes determinantes da supressão imune nestas lesões. ${ }^{1,11}$ Os mecanismos envolvidos neste processo ainda não são completamente conhecidos, mas estudos in vitro têm demonstrado que os MATs são capazes de inibir a proliferação de linfócitos T CD8+, um evento que pode ocorrer independentemente do metabolismo da L-arginina e que parece envolver a família de moléculas B7. ${ }^{18,19}$ Além disso, tem sido revelado um envolvimento importante dos MATs M2 no recrutamento de células Treg para o microambiente tumoral, particularmente por meio da secreção de quimiocinas, como a CCL20 e a CCL22. ${ }^{20}$

Os macrófagos têm sido identificados como um componente importante do infiltrado inflamatório de vários tumores malignos, como carcinomas de fígado, mama e próstata, gliomas e linfomas. Em muitos destes tumores, o aumento de macrófagos no infiltrado inflamatório tem sido correlacionado com um pobre prognóstico. ${ }^{7,15,21,22,23}$ Em relação ao CCE oral, pesquisas sobre a presença e a possível influência dos MATs no comportamento biológico desta neoplasia são escassas e foram publicadas, em sua maioria, apenas nos últimos oito anos. $5,6,7,8,9,24,25,26,27,28,29$

Em um estudo imuno-histoquímico sobre a composição do infiltrado inflamatório peritumoral em trinta CCEs orais, Vieira et al. ${ }^{24}$ observaram que os linfócitos $\mathrm{T}$ eram os tipos celulares mais numerosos, com predomínio das células T CD8+ sobre as células T CD4+, seguidos dos linfócitos B e macrófagos (CD68), com uma menor proporção de neutrófilos. Para os autores, o grande número de linfócitos $\mathrm{T}$ e macrófagos observados no infiltrado inflamatório peritumoral sugere que a resposta imune celular é o principal mecanismo de defesa contra o CCE oral.

El-Rouby ${ }^{5}$ analisou, por imunohistoquímica, a presença de MATs (CD68) e 
a densidade microvascular em 32 casos de CCEs orais. Por meio de análise de regressão linear, foi constatada uma correlação positiva entre a quantidade de MATs e a densidade microvascular nestas lesões. Além disso, foi observado um aumento significativo na quantidade de MATs, desde os carcinomas bem diferenciados até os carcinomas pobremente diferenciados. Para o autor, os MATs exerceriam um importante papel na regulação da angiogênese nos CCEs orais.

Por sua vez, Lu et al. ${ }^{6}$ avaliaram, por imunohistoquímica, a presença de MATs (CD68) em 92 CCEs orais e evidenciaram um aumento significativo nas quantidades destes tipos celulares em tumores de maiores dimensões, bem como em casos com metástase linfonodal e com estadiamento clínico avançado. Além disso, pacientes com tumores exibindo maiores contagens de macrófagos apresentavam menor sobrevida livre de doença e menor sobrevida geral. Para os autores, os MATs podem constituir um importante indicador de prognóstico nos CCEs orais.

Em um estudo imuno-histoquímico, Bôas et al. $^{8}$ avaliaram uma possível correlação entre o índice de proliferação das células neoplásicas, a quantidade de MATs (CD68) e a expressão de ciclooxigenase-2, em 27 CCEs orais. Apesar de terem sido identificados MATs em todos os casos estudados, os autores não evidenciaram correlação entre a quantidade destes tipos celulares e os demais parâmetros analisados. Com base no importante papel dos macrófagos na progressão tumoral, Bôas et al. não descartam a participação dos MATs na carcinogênese oral, provavelmente por meio da secreção de moléculas envolvidas no processo de remodelação da MEC, como as MMPs.

Em uma amostra de 108 casos de CCEs orais, Fujii et al. ${ }^{9}$ avaliaram a presença de MATs por imuno-histoquímica. Por meio de análises de Kaplan-Meier, os autores constataram correlação significativa entre altos níveis de MATs M2 (CD163) e menores taxas de sobrevida dos pacientes. Além disso, após análise multivariada de sobrevida, o alto nível de MATs M2 nos CCEs orais apresentouse como fator prognóstico independente.

Por meio de um estudo imuno-histoquímico, Ferreira et al. ${ }^{25}$ avaliaram a relação da expressão da quimiocina CCL2 pelas células tumorais e estromais com parâmetros clínicopatolológicos em CCEs de lábio e CCEs intraorais. Os autores constataram que os casos de CCEs de lábio apresentavam menor percentual de células estromais imunopositivas para quimiocina CCL2 e maiores quantidades de MATs (CD68) em comparação aos CCEs intraorais. De acordo com esses autores, o comportamento menos agressivo dos CCEs de lábio poderia estar relacionado à infiltração de macrófagos por mecanismos não mediados pela quimiocina CCL2.

Fujita et al. ${ }^{28}$ avaliaram a relação entre os níveis séricos e intratumorais de IL-8 e diversos parâmetros clínico-patológicos em casos de CCE oral. Os autores encontraram uma forte correlação entre os níveis de IL-8, tanto séricos $(p=0,038)$ como intratumorais ( $p=0,0003)$, e a expressão de CD163+ no front invasivo de CCEs orais. Além disso, esses autores observaram in vitro que a IL-8 aumentava significativamente o número de células CD163+, sugerindo que esta interleucina poderia induzir a polarização de macrófagos M2 no CCE oral.

Recentemente, $\mathrm{He}$ et al. ${ }^{27}$ investigaram a relação entre MATs (CD68+ e CD163+), marcadores de células tronco tumorais (CSC) (ALDH1, CD44 e SOX2) e parâmetros clínicopatológicos, por meio de tissue microarray em 16 espécimes de mucosa oral normal, seis de displasia epitelial e 43 de CCE oral. Foi verificado que a quantidade de MATs, bem como a expressão dos CSCs, aumentou progressivamente da mucosa oral normal ao 
CCE. Aquantidade de MATs revelou associação significativa com metástase linfonodal $(p<$ $0,05)$ e correlação com os marcadores de CSCs. Além disso, a análise de Kaplan-Meier revelou que os casos que superexpressavam CD163 apresentavam pior sobrevida global ( $p$ $<0,05)$. Para os autores, os MATs poderiam ser considerados marcadores do comportamento biológico mais agressivo em CCE oral.

\section{Discussão}

As populações celulares e os mediadores químicos presentes no microambiente tumoral podem contribuir para a progressão das neoplasias ou desempenhar atividades antitumorais. Os MATs são os principais e mais abundantes componentes celulares no microambiente de várias neoplasias malignas, incluindo o CCE oral. 5,8,26,27,28,29 Neste último, os estudos têm sugerido um importante papel para os MATs, especialmente aqueles com o fenótipo $M 2$, nos processos de invasão e metástase. Além disso, a presença desses tipos celulares no CCE oral tem sido associada à diminuição da sobrevida dos pacientes e a piores prognósticos. . $, 9,26,27,28,29$

Embora incompletamente conhecidos, foram propostos diversos mecanismos pelos quais os MATs M2 auxiliariam no crescimento e na progressão tumoral, incluindo a modulação da proliferação celular, da angiogênese, da remodelação da MEC e da resposta imune. ${ }^{1}$ No contexto do CCE oral, os resultados do estudo de Costa et al. ${ }^{26}$ enaltecem a participação dos MATs na modulação da resposta imune. Esses autores avaliaram e caracterizaram o fenótipo de populações de macrófagos (M1/ M2), por meio da expressão de citocinas anti (IL-10 e TGF- $\beta$ ) e pró-inflamatórias (IL-12, IL-23 e IFN- $\gamma$ ) em CCEs orais e em mucosa oral normal por imuno-histoquímica, citometria de fluxo e PCR quantitativo (qRT-PCR). Foi constatado um predomínio de MATs M2 no microambiente dos CCEs orais, caracterizado pela maior proporção de células que expressavam simultaneamente as citocinas anti-inflamatórias. Além disso, verificou-se uma proporção significativamente maior de macrófagos (CD68) no grupo de CCE oral com metástase quando comparado ao grupo sem metástase $(p=0,038)$. Com base nos achados, os autores sugerem que os MATs M2 podem favorecer o desenvolvimento de metástases no CCE oral e, consequentemente, reduzir a sobrevida dos pacientes, provavelmente por meio da imunossupressão local via produção de TGF- $\beta$.

A despeito dos achados destacados anteriormente, pesquisas realizadas com o intuito de analisar possíveis relações entre a quantidade de MATs, a proliferação celular e a angiogênese em CCEs orais têm demonstrado resultados conflitantes. Ferreira et al. ${ }^{25}$, em estudo com CCEs de cavidade oral e de lábio, observaram uma relação estatisticamente significativa entre menores quantidades de MATs (CD68) e maiores índices de proliferação das células neoplásicas. Por sua vez, Bôas et al. $^{8}$ não observaram correlação entre a quantidade de MATs (CD68), o índice de proliferação das células neoplásicas e a expressão de ciclooxigenase-2 em CCEs orais. Por outro lado, El-Rouby ${ }^{5}$ constatou uma correlação positiva entre a quantidade de MATs (CD68) e a densidade microvascular nos CCEs orais. Tais resultados divergentes podem estar relacionados à avaliação dos MATs nesses estudos imuno-histoquímicos apenas com o anticorpo anti-CD68. Sabe-se que esse antígeno pode ser expresso tanto por MATs M1 como por MATs M2. ${ }^{10,30}$

Os estudos existentes, apesar de escassos, identificam os macrófagos como um componente importante do microambiente dos CCEs orais. . $, 6,7,8,9,24,25,26,27,28,29$ No entanto, os mecanismos pelos quais os macrófagos são recrutados para esse microambiente, bem como os mediadores químicos envolvidos na 
determinação do estado de polarização desses tipos celulares nos CCE orais, ainda são pouco conhecidos. Neste sentido, merecem destaque os achados reportados por Ferreira et al. ${ }^{25} \mathrm{e}$ Fujita et al. ${ }^{28}$. Ferreira et al. ${ }^{25}$ constataram que CCEs de lábio, em comparação aos CCEs de cavidade oral, apresentavam menor percentual de células estromais imunopositivas para quimiocina CCL2 e maiores quantidades de MATs (CD68). Estes autores sugeriram que a infiltração de macrófagos por mecanismos não mediados pela quimiocina CCL2 poderia ser um fator determinante do comportamento biológico menos agressivo dos CCEs de lábio. Por sua vez, Fujita et al. ${ }^{28}$ observaram in vitro que a IL-8 aumentava consideravelmente o número de células CD163+, sugerindo que esta interleucina poderia induzir a polarização de macrófagos M2 no CCE oral.

A infiltração de macrófagos M2 apenas em estágios mais avançados da carcinogênese oral é outro aspecto que tem sido enaltecido na literatura, sugerindo uma participação importante destas células no processo de invasão dos tecidos circunvizinhos. Corroborando esta sugestão, estudos realizados recentemente revelaram que lesões com displasia epitelial|,29 e carcinomas in situ ${ }^{29}$ raramente apresentam estes componentes celulares, estando o aparecimento de quantidades consideráveis dos macrófagos M2 apenas em lesões de CCE oral. De acordo com Fujii et al., ${ }^{9}$ quando há uma interação dessas células com fibroblastos associados a tumores, há uma maior produção de citocinas e fatores angiogênicos no microambiente tumoral, favorecendo o processo de transformação maligna.

\section{Conclusão}

Os macrófagos constituem um componente importante do microambiente do CCE oral. A maior quantidade de MATs pode estar associada a um comportamento biológico mais agressivo do CCE oral e, consequentemente, à diminuição da sobrevida dos pacientes. Neste contexto, os macrófagos M2 assumem posição de destaque, em virtude de sua capacidade de modular a proliferação celular, a angiogênese, a remodelação da MEC e a resposta imune. Em decorrência do número relativamente pequeno de estudos sobre o assunto, faz-se necessário o desenvolvimento de pesquisas que esclareçam os mecanismos exatos da polarização dos MATs e como eles são capazes de influenciar o comportamento biológico do CCE oral. O entendimento desses mecanismos poderá auxiliar na compreensão dos processos neoplásicos, com potencial para utilização no desenvolvimento de imunoterápicos e quimioterápicos contra o CCE oral.

\section{RefERÊNCIAS}

1 Heusinkveld M, Van Der Bur SH. Identification and manipulation of tumor associated macrophages in human cancer. J Transl Med. 2011; 9(1): 216.

2 Lumeng CN, Saltiel AR. Inflammatory links between obesity and metabolic disease. J Clin Invest. 2011; 121(6): 2111-7.

3 Hao NB, Lü MH, Fan YH, Cao YL, Zhang $Z R$, Yang SM. Macrophages in tumor microenvironments and the progression of tumors. Clin Dev Immunol. 2012; 2012:948098.

4 Sica A, Larghi P, Mancino A, Rubino L, Porta C, Totaro MG, et al. Macrophage polarization in tumour progression. Semin Cancer Biol. 2008; 18(5): 349-55.

5 El-Rouby DH. Association of macrophages with angiogenesis on oral verrucous and squamous cell carcinoma. J Oral Pathol Med. 2010; 39(7): 559-64.

6 Lu CF, Huang CS, Tjiu JW, Chiang CP. Infiltrating macrophage count: A significant predictor for the progression and prognosis of oral squamous cell carcinomas in Taiwan. Head Neck. 2010; 32(1): 18-25.

7 Laoui D, Movahedi K, Van Overmeire E, Van der Bossche J, Schouppe E, Mommer C, et al. Tumor-associated macrophages in breast 
cancer: distinct subsets, distinct functions. Int J Dev Biol. 2011; 55(7-9): 861-7.

8 Bôas DS, Takiya CM, Coelho-Sampaio TL, Monção-Ribeiro LC, Ramos EA, Cabral MG, et al. Immunohistochemical detection of Ki67 is not associated with tumor-infiltrating macrophages and cyclooxygenase- 2 in oral squamous cell carcinoma. J Oral Pathol Med. 2010; 39(7): 565-70.

9 Fujii N, Shomori K, Shiomi T, Nakabayashi M, Takeda C, Ryoke K, et al. Cancer-associated fibroblasts and CD163-positive macrophages in oral squamous cell carcinoma: their clinicopathological and prognostic significance. J Oral Pathol Med. 2012; 41(6): 444-51.

$10 \mathrm{Ma} \mathrm{J}$, Liu L, Che G, Yu N, Dai F, You Z. The M1 form of tumor-associated macrophages in non-small cell lung cancer is positively associated with survival time. BMC Cancer. 2010; 10(112): 1-9.

11 Baay M, Brouwer A, Pauwels P, Peeters M, Lardon F. Tumor cells and tumor-associated macrophages: secreted proteins as potential targets for therapy. Clin Develop Immunol. 2011; 2011(1): 565187.

12 Mantovani A, Germano G, Marchesi F, Locatelli M, Biswas SK. Cancer-promoting tumorassociated macrophages: New vistas and open questions. Eur J Immunol. 2011; 41(1): 2522-5.

13 Davies LC, Jenkins SJ, Allen JE, Taylor PR. Tissue-resident macrophages. Nat Immunol. 2013;14(10): 986-95.

14 Tay SS, Roediger B, Tong PL, Tikoo S, Weninger W. The skin-resident immune network. Curr Dermatol Rep. 2013; 3(1): 13-22.

15 Mantovani A, Sozzani S, Locati M, Allavena $P$, Sica A. Macrophage polarization: Tumorassociated macrophages as a paradigm for polarized M2 mononuclear phagocytes. Trends Immunol. 2002; 23(11): 549-55.

16 Mason SD, Joyce JA. Proteolytic networks in cancer. Trends Cell Biol. 2011; 21(4): 228-37.

17 Reilkoff RA, Bucala R, Herzog EL. Fibrocytes: emerging effector cells in chronic inflammation. Nat Rev Immunol. 2011; 11(6): 427-35.

18 Kryczek I, Zou L, Rodriguez P, Zhu G, Wei S, Mottram P, et al. B7-H4 expression identifies a novel suppressive macrophage population in human ovarian carcinoma. J Exp Med. 2006; 203(4): 871-81.

19 Kuang DM , Zhao Q, Peng C, Xu J, Zhang JP, Wu $\mathrm{C}$, Zheng L. Activated monocytes in peritumoral stroma of hepatocellular carcinoma foster immune privilege and disease progression through PDL1. J Exp Med. 2009; 206(6): 1327-37.

20 Liu J, Zhang N, Li Q, Zhang W, Ke F, Leng Q, et al. Tumor-associated macrophages recruit CCR6+ regulatory $T$ cells and promote the development of colorectal cancer via enhancing CCL20 production in mice. PLoS One. 2011; 29;6(4): e19495.

21 Lissbrant IF, Stattin P, Wikstrom P, Damber JE, Egevad L, Bergh A. Tumor associated macrophages in human prostate cancer: relation to clinicopathological variables and survival. Int J Oncol. 2000; 17(3): 445-51.

22 Bingle L, Brown NJ, Lewis CE. The role of tumorassociated macrophages in tumor progression: implications for new anticancer therapies. J Pathol. 2002; 196(3): 254-65.

23 Dave SS, Wright G, Tan B, Rosenwald A, Gascoyne RD, Chan WC, et al. Prediction of survival in follicular lymphoma based on molecular features of tumor-infiltrating immune cells. N Engl J Med. 2004; 351(1): 2159-69.

24 Vieira FL, Vieira BJ, Guimaraes MA, Aarestrup FM. Cellular profile of the peritumoral inflammatory infiltrate in squamous cell carcinomas of oral mucosa: correlation with the expression of Ki67 and histological grading. BMC Oral Health. 2008; 8: 25.

25 Ferreira FO, Ribeiro FL, Batista AC, Leles CR, Alencar RCG, Silva TA. Association of CCL2 with lymph node metastasis and macrophage infiltration in oral cavity and lip squamous cell carcinoma. Tumor Biol. 2008; 29(3): 114-21.

26 Costa NL, Valadares MC, Souza PPV, Mendonça EF, Oliveira JC, Silva TA, et al. Tumor-associated macrophages and the profile of inflammatory cytokines in oral squamous cell carcinoma. Oral Oncol. 2013; 49(3): 216-23.

27 He KF, Zhang L, Huang CF, Ma SR, Wang YF, Wang WM, et al. CD163+ tumor associated macrophages correlated with prognosis and cancer stem cells in oral squamous cell carcinoma. Biomed Res Int. 2014; 2014: 838632. 
28 Fujita Y, Okamoto M, Goda H, Tano T, Nakashiro K, Sugito A, et al. Prognostic significance of interleukin-8 and CD163positive cell infiltration in tumor tissues in patients with oral squamous cell carcinoma. PLoS One. 2014; 29(12): e110378.

29 Wang S, Sun M, Gu C, Wang X, Chen D, Zhao E, et al. Expression of CD163, interleukin-10, and interferon-gamma in oral squamous cell carcinoma: mutual relationships and prognostic implications. Eur J Oral Sci. 2014; 122(3): 202-9. 30 Wada DA, Wilcox RA, Weenig RH, Gibson LE. Paucity of intraepidermal FoxP3-positive T cells in cutaneous T-cell lymphoma in contrast with spongiotic and lichenoid dermatitis. J Cutan Pathol. 2010; 37(5): 535-41.

Submetido em: 18-2-2015

Aceito em: 25-3-2015 